\title{
Different patterns of nigrostriatal degeneration in tremor type versus the akinetic-rigid and mixed types of Parkinson's disease at the early stages: Molecular imaging with ${ }^{123}$ I-FP-CIT SPECT
}

\author{
O. SCHILLACI ${ }^{1,2}$, A. CHIARAVALLOTI ${ }^{1}$, M. PIERANTOZZI ${ }^{3,4}$, B. DI PIETRO ${ }^{1}$,

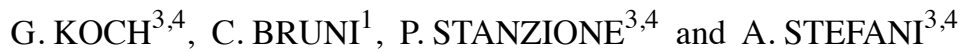 \\ ${ }^{1}$ Department of Biopathology and Diagnostic Imaging, University Tor Vergata, Rome; ${ }^{2}$ IRCCS Neuromed, Pozzilli; \\ ${ }^{3}$ Department of Neurology, University Tor Vergata, Rome; ${ }^{4}$ IRCCS Fondazione Santa Lucia, Rome, Italy
}

Received May 26, 2011; Accepted July 1, 2011

DOI: $10.3892 / \mathrm{ijmm} .2011 .764$

\begin{abstract}
The various associations of motor and non-motor symptoms, the onset of motor complications, the cognitive disorder's appearance and other factors make Parkinson's disease (PD) a heterogeneous syndrome with multiple phenotypes. The necessity of discriminating between different forms of PD could have a role in understanding the pathophysiology of extrapyramidal signs with clinical implications. The aim of this study was to evaluate if there is a relationship between the clinical motor phenotypes of PD and the scintigraphic pattern of ${ }^{123}$ I-FP-CIT single photon emission computed tomography (SPECT). We examined 47 patients with early idiopathic PD (25 males; 22 females; mean age 58 \pm 2 years) and subdivided them in different clinical forms on the basis of dominance of resting tremor $(n=20)$, bradykinesia plus rigidity $(n=20)$ and the presence of both clinical signs [mixed type $(M T, n=7)]$. We correlated this status with the semi-quantitative analysis of SPECT with ${ }^{123}$ I-FP-CIT. Tremor type patients showed a lower reduction of ${ }^{123}$ I-FP-CIT uptake compared to akineticrigid type patients in contralateral caudate $(\mathrm{P}=0.0139)$ and putamen $(\mathrm{P}=0.0028)$ nuclei. ${ }^{123} \mathrm{I}-\mathrm{FP}-\mathrm{CIT}$ uptake was higher in the ipsilateral caudate $(\mathrm{P}=0.0050)$ and putamen $(\mathrm{P}=0.0012)$ of tremor type patients compared to akinetic-rigid type patients. Comparisons of the striatal uptake in the tremor type and akinetic-rigid type patients with the MT patients revealed significant differences only in the ipsilateral and contralateral caudate. Our data indicate that in akinetic-rigid patients the dopaminergic system is more involved compared to that in the tremor type patients and that this difference is present from the initial stage of the disease. Moreover, our results suggest that
\end{abstract}

Correspondence to: Dr Orazio Schillaci, Department of Biopathology and Diagnostic Imaging, University Tor Vergata, Viale Oxford 81, I-00173 Rome, Italy

E-mail: orazio.schillaci@uniroma2.it

Key words: DAT SCAN, Parkinson's disease, tremor type, akineticrigid type, mixed type
PD phenotypes could be related not only to the dopaminergic involvement but also to other systems.

\section{Introduction}

The relationships between movement disorders and the dopaminergic system have been extensively investigated, both in terms of presynaptic and postsynaptic processes (1). The particular interest in the dopamine active transporter (DAT) is related to the assessment of dopaminergic neuron loss in Parkinson's disease (PD) $(2,3)$ and movement disorders $(2,4,5)$. ${ }^{123}$ I-FP-CIT is a radiopharmaceutical highly selective for the DAT on the presynaptic processes of the nigrostriatal neurons (6). DAT is located on the plasma membrane of nerve terminals in a small number of neurons in the brain, especially in the striatum and nucleus accumbens, but also in the globus pallidus, cingulate cortex, olfactory tubercle, amygdala and midbrain (7).

In idiopathic PD (IPD), 50\% of patients complain of slowing up at presentation. The slowness of IPD involves both initiation and execution of movements, particularly sequential and volitional actions (8). About $40 \%$ of patients will complain of tremulousness of hands at rest, which improves with action and $80 \%$ of them will have an asymmetrical $3-5 \mathrm{~Hz}$ rest tremor evident on examination (9). A variant of IPD based on the clinical course is the mixed type (MT) with the presence of both bradykinesia and resting tremor. In concordance to their differences in brain biochemical abnormalities, the three subtypes of IPD have different courses (10).

In this study, we compared ${ }^{123}$ I-FP-CIT uptake in patients with different subtypes of early PD, i.e. Hoen and Yahr (H\&Y) stages 1 and 1.5, in order to assess the relationship between clinical onset and nigrostriatal degeneration.

\section{Materials and methods}

Patients. The study involved 47 patients with IPD; $35 \mathrm{H} \& \mathrm{Y}$ stage 1 and 12 stage 1.5, 25 males and 22 females, mean age $58 \pm 2$ years. A clinical examination was conducted by one experienced neurologist (P.S.) and PD was diagnosed using United Kingdom Parkinson's Disease Society Brain Bank 
Table I. General overview of the population examined.

\begin{tabular}{|c|c|c|c|c|}
\hline & Population $(n=47)$ & TDT $(n=20)$ & $\operatorname{ART}(n=20)$ & $\operatorname{MT}(n=7)$ \\
\hline Age of onset, years \pm SD & $58 \pm 2$ & $56 \pm 10$ & $59 \pm 11$ & $59 \pm 9$ \\
\hline \multicolumn{5}{|l|}{ Gender } \\
\hline Male & 25 & 11 & 10 & 4 \\
\hline Female & 22 & 9 & 10 & 3 \\
\hline Disease duration, months \pm SD & & $19 \pm 12$ & $17 \pm 7$ & $21 \pm 13$ \\
\hline UPDRS III, mean \pm SD & & $14 \pm 6$ & $15 \pm 7$ & $17 \pm 5$ \\
\hline \multicolumn{5}{|l|}{ Hoehn and Yahr } \\
\hline 1 & 36 & 16 & 15 & 5 \\
\hline 1.5 & 11 & 4 & 5 & 2 \\
\hline
\end{tabular}

TDT, tremor-dominant type; ART, akinetic-rigid type; MT, mixed type patients; UPDRS III, UPDRS motor examination section score.

(UKPDSBB) criteria (11). The motor part of the UKPDSBB was used to assess the severity of disease (12). On the basis of the predominant motor features in the Unified Parkinson's Disease rating scale (UPDRS), patients were subtyped into 1 of 3 clinical groups, namely tremor-dominant type (TDT), akinetic-rigid type (ART) and MT, following the methods previously proposed (13-15). Mini mental state examination (MMSE) did not reveal any cognitive deficit. Twenty patients featured TDT, 20 ART and 7 MT. We calculated a 'tremor score' and a 'non-tremor score' for each patient $(14,15)$. The data concerning the clinical subtypes of patients with PD are reported in Table I. The tremor score was derived from the sum of UPDRS items 20 (tremor at rest) and 21 (action or postural tremor of hands) divided by 7 (the number of the single sub items included). The non-tremor score was derived from the sum of UPDRS items 18 (speech), 19 (facial expression), 22 (rigidity), 27 (arising from the chair), 28 (posture), 29 (gait), 30 (postural stability) and 31 (body bradykinesia and hypokinesia) divided by 12 (the number of the single sub-items included). The patient was classified as TDT, if the tremor score was at least twice the non-tremor score. The remaining patients, in whom the tremor and non-tremor score differed by less than a factor of 2 , were classified as MT.

Data acquisition. Each patient was i.v. injected with $185 \mathrm{MBq}$ of ${ }^{123} \mathrm{I}$-FP-CIT (Amersham Health, UK) that was given at the same time of the day and under the same experimental conditions. Perchlorate $(1000 \mathrm{mg})$ was administered at least 30 min before radiopharmaceutical injection to block thyroid uptake of free radioactive iodine (16). Imaging was always performed $4 \mathrm{~h}$ after ${ }^{123}$ I-FP-CIT using a dual-head gamma camera (Millennium VG; General Electric Medical Systems, Milwaukee, WI), equipped with low-energy high-resolution collimators. Single photon emission computed tomography (SPECT) studies were acquired using the following parameters: $128 \times 128$ matrix, 120 projections (rotation of $360^{\circ} \mathrm{C}$ ), $40 \mathrm{sec} /$ projection. The slice thickness was $4.42 \mathrm{~mm}$. Reconstruction was performed by filtered back-projection with a Butterworth filter (cut-off frequency 0.5 , order 10) to produce transaxial slices that were attenuation corrected. Attenuation correction was performed according to Chang's method (17) using a coefficient $\mu=0.11 / \mathrm{cm}$, after manually drawing an ellipse around the head contour. For analysis of ${ }^{123}$ I-FP-CIT striatal uptake, the ratio of specific to non-specific binding was calculated by summing the three adjacent transverse slices that showed the most intense striatal uptake. The slices were re-oriented to be parallel to the canthomeatal line. A standard ROI template, constructed manually according to a stereotactic atlas as previously described by Booij et al (18) and including fixed regions for both putamina, caudate nuclei and occipital cortices, was placed on the summed images. Small variations in the individual brain required movement of the ROIs within the template, without changing their size or shape, for optimal positioning. Therefore, the exact same ROIs were used in all patients for both images. The ratio of specific to non-specific binding was then calculated as ${ }^{123}$ I-FP-CIT binding=(ROI$\mathrm{O}) / \mathrm{O}$, in which ROI represents the mean counts in the region of interest (putamen or caudate nucleus) and $\mathrm{O}$ represents the mean counts in the occipital cortex (19).

Statistical analysis. We calculated the means and standard deviation for age, UPDRS motor examination score (UPDRS III), H\&Y grade, disease duration and the results of the semiquantitative analysis for the ipsi and contralateral putamen and caudate nucleus. To detect differences of the dopaminergic deficit between the sub groups (semi-quantitative analysis, non-parametric data) we used Mann-Withney test. Two-way ANOVA was used in order to assess a relationship between $\mathrm{H} \& \mathrm{Y}$ stage and disease phenotype. A hypothesis was considered valid when P-value $\leq 0.05$.

\section{Results}

Differences in gender, disease duration, age and UPDRS between patients with ART, TDT and MT were not significant; we did not find statistical significant differences in $\mathrm{H} \& \mathrm{Y}$ stages and the patient's phenotype. Patient population characteristics are summarized in Table I.

The results of the semi-quantitative analysis of SPECT data are summarized in Table II. The average uptake value 
Table II. Mean and SD of the striatal ${ }^{123}$ I-FP-CIT uptake in clinical subtypes.

\begin{tabular}{|c|c|c|c|c|c|}
\hline & TDT $(n=20)$ & $\operatorname{ART}(\mathrm{n}=20)$ & $\operatorname{MT}(\mathrm{n}=7)$ & Comparisons & P-value \\
\hline Contralateral caudate & $2.97 \pm 0.67$ & $2.46 \pm 0.67$ & $2.17 \pm 0.37$ & $\begin{array}{l}\text { TDT vs. ART } \\
\text { TDT vs. MT } \\
\text { ART vs. MT }\end{array}$ & $\begin{array}{l}0.0139 \\
0.0466 \\
0.5160\end{array}$ \\
\hline Ipsilateral caudate & $3.11 \pm 0.60$ & $2.56 \pm 0.73$ & $2.57 \pm 0.64$ & $\begin{array}{l}\text { TDT vs. ART } \\
\text { TDT vs. MT } \\
\text { ART vs. MT }\end{array}$ & $\begin{array}{l}0.0050 \\
0.0434 \\
0.7070\end{array}$ \\
\hline Contralateral putamen & $2.23 \pm 0.42$ & $1.79 \pm 0.45$ & $1.88 \pm 0.42$ & $\begin{array}{l}\text { TDT vs. ART } \\
\text { TDT vs. MT } \\
\text { ART vs. MT }\end{array}$ & $\begin{array}{l}0.0028 \\
0.0676 \\
0.5060\end{array}$ \\
\hline Ipsilateral putamen & $2.47 \pm 0.44$ & $1.90 \pm 0.50$ & $2.21 \pm 0.46$ & $\begin{array}{l}\text { TDT vs. ART } \\
\text { TDT vs. MT } \\
\text { ART vs. MT }\end{array}$ & $\begin{array}{l}0.0012 \\
0.2451 \\
0.1055\end{array}$ \\
\hline
\end{tabular}

A Contralateral caudate FP-CIT uptake

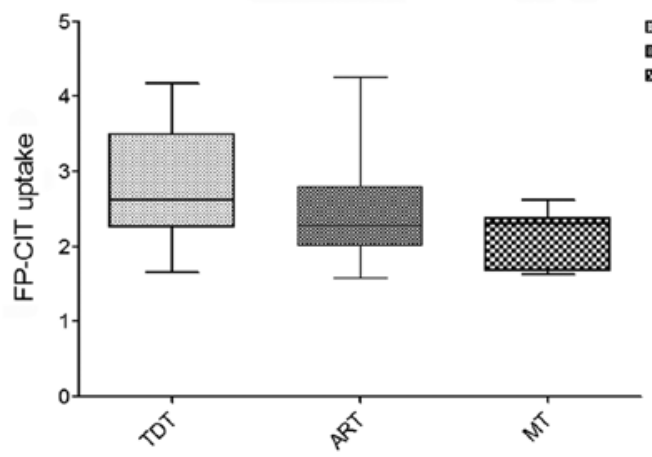

C Ipsilateral caudate FP-CIT uptake

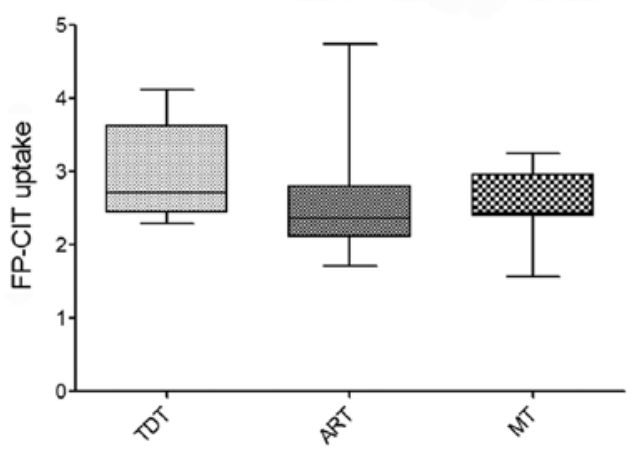

B Contralateral putamen FP-CIT uptake
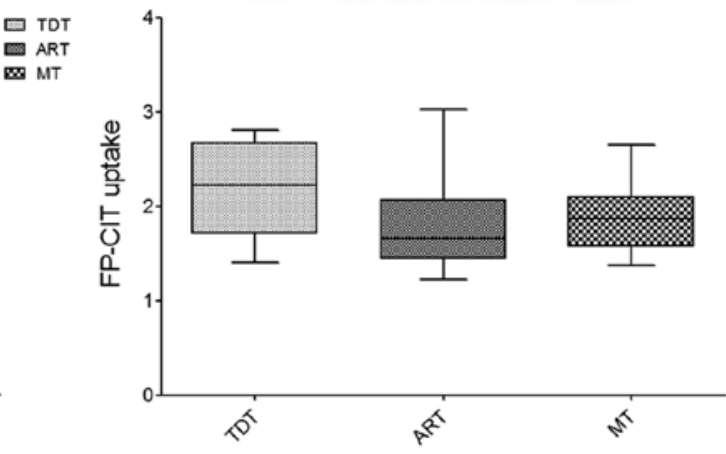

D Ipsilateral putamen FP-CIT uptake
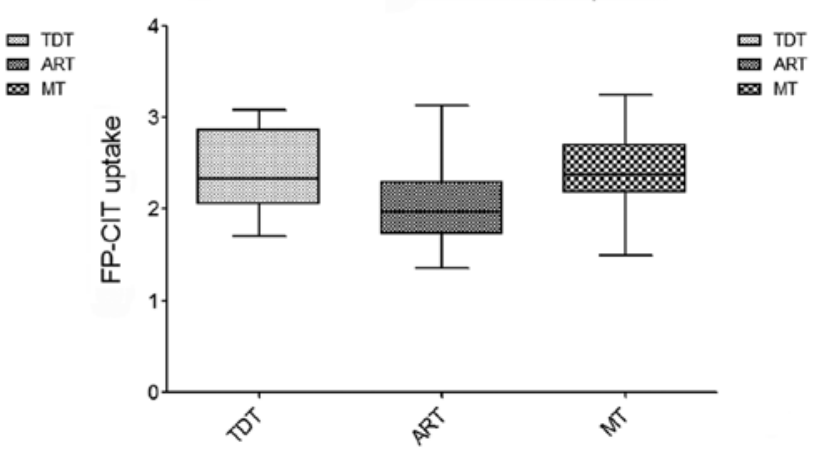

Figure 1. FP-CIT uptake in both contralateral and ipsilateral putamen and caudate. The dopaminergic system is more involved in ART and MT patients in both the contralateral and ipsilateral caudate in comparison with MT patients (A and C). TDT patients present a higher ${ }^{123}$ I-FP-CIT uptake in the contralateral and ipsilateral putamen in comparison with ART patients (B and D).

of contralateral and ipsilateral caudate was $2.97 \pm 0.67$ and $3.11 \pm 0.60$ in TDT; $2.46 \pm 0.67$ and $2.56 \pm 0.73$ in ART. In MT they were $2.17 \pm 0.37$ and $2.57 \pm 0.64$, respectively. In the contralateral and ipsilateral putamen, the average ${ }^{123} \mathrm{I}-\mathrm{FP}-\mathrm{CIT}$ uptake was $2.23 \pm 0.42$ and $2.47 \pm 0.44$ in TDT; $1.79 \pm 0.45$ and $1.90 \pm 0.50$ in ART; and $1.88 \pm 0.42$ and $2.21 \pm 0.46$ in MT (Table II).

In TDT patients, the ${ }^{123}$ I-FP-CIT uptake in the caudate contralateral to the side affected was higher in comparison with that in the ART $(\mathrm{P}=0.0139)$ and MT $(\mathrm{P}=0.0466)$ types while no differences were found when comparing ART patients with MT ( $\mathrm{P}=0.5160)$ patients (Fig. 1).

In the contralateral putamen, we found a higher ${ }^{123} \mathrm{I}-\mathrm{FP}-\mathrm{CIT}$ uptake in TDT vs. ART $(\mathrm{P}=0.0028)$ patients, while we did not identify statistically significant differences when comparing the contralateral putamen uptake in the TDT vs. the MT types $(\mathrm{P}=0.0676)$. Comparison of the ${ }^{123} \mathrm{I}-\mathrm{FP}-\mathrm{CIT}$ uptake at this level, identified non-significant differences between ART and MT (P=0.5060) (Fig. 1). 

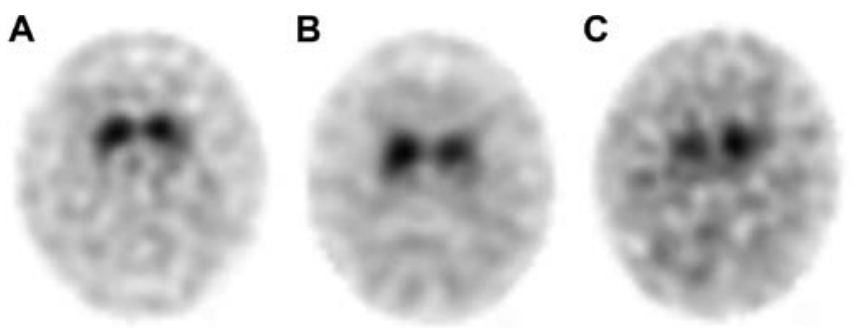

Figure 2. Sample images of ${ }^{123}$ I-FP-CIT SPECT: (A) a TDT patient, (B) an ART patient and (C) an MT patient.

In reference to the ipsilateral caudate, we found a higher ${ }^{123} \mathrm{I}-\mathrm{FP}-\mathrm{CIT}$ uptake in TDT than ART $(\mathrm{P}=0.0050)$ and MT $(\mathrm{P}=0.0434)$ patients. We did not find statistically significant differences in the ipsilateral caudate uptake between ART and MT $(\mathrm{P}=0.7070)$ types. ${ }^{123} \mathrm{I}-\mathrm{FP}-\mathrm{CIT}$ uptake was higher in the ipsilateral putamen of TDT than in ART patients $(\mathrm{P}=0.0012)$. Differences observed in the ipsilateral putamen uptake between TDT and MT patients $(\mathrm{P}=0.2451)$ and between ART and MT $(\mathrm{P}=0.1055)$ were not statistically significant (Fig. 1). Sample images of DAT SCAN in a TDT patient, ART patient and MT patient are shown in Fig. 2.

\section{Discussion}

IPD is a degenerative disorder of the nervous system in which mainly the presynaptic dopaminergic neurons are affected: the dopamine synthesis as well as the active transport of dopamine into the synaptic gap by presynaptic DATs is reduced. First parkinsonian symptoms occur when the concentration of dopamine within the basal ganglia is reduced by at least $80 \%$ (20). The reduced DAT density represents a typical phenomenon of IPD: it can be measured by SPECT with the radiopharmaceutical ${ }^{123} \mathrm{I}-\mathrm{FP}-\mathrm{CIT}$ that has a high affinity to presynaptic DAT (20). PD patients typically show a significantly lower striatal FP-CIT uptake than healthy controls, and therefore SPECT supports the diagnosis of IPD (21).

Rigidity, akinesia-hypokinesia-bradykinesia, and resting tremor are main motor features of IPD: some patients are tremor dominant some are akinetic-rigid, while the severity of these symptoms is comparable in others. Previous studies reported different findings between TDT and ART of PD patients; PD progresses faster in ART patients than in TDT patients, and ART patients exhibit more depressive symptoms than TDT patients $(14,22)$.

In the present study, we used ${ }^{123}$ I-FP-CIT SPECT to verify the possible differences in the uptake among various IPD subtypes. In fact, molecular imaging using ${ }^{123}$ I-FP-CIT SPECT proved to be a helpful diagnostic method for the evaluation of patients with movement disorders. If one considers DAT a surrogate marker of dopaminergic nigrostriatal neurons, ${ }^{123}$ I-FP-CIT imaging of the DAT sites can identify nigrostriatal dopaminergic deficit in IPD patients in vivo. Several SPECT studies have demonstrated high accuracy in differentiating patients with IPD and parkinsonian syndromes from patients with essential tremor and healthy controls (21). In particular, when clinical features are less clear, as is often the case in early presentation, ${ }^{123}$ I-FP-CIT scanning is able to aid in the diagnostic process (23).
Our results indicate that striatal ${ }^{123}$ I-FP-CIT uptake was less impaired in TDT patients than in ART or MT patients at the same stage of the disease. Already, Spiegel et al (15) reported a significantly higher ${ }^{123}$ I-FP-CIT uptake in TDT patients vs. ART ones in putamen and caudate nucleus contralateral to the clinically more affected body side as well as putamen and caudate nucleus ipsilateral to the clinically more affected body side. These findings have been confirmed by our present evaluation; moreover similar outcomes were disclosed between ART and MT patients: there were no significant differences concerning binding properties both in the contralateral or ipsilateral caudate nucleus and in the contralateral or ipsilateral putamen. Nevertheless, in contrast to our study, in their series TDT patients revealed a significantly higher ${ }^{123}$ I-FP-CIT uptake in all four striatal areas examined than MT patients, whereas we found these differences statistically significant only for caudate nuclei but not for the contralateral and ipsilateral putamen. Comparing putamen uptake with that in the caudate, in our study in all subgroups (TDT, ART and MT) putamen uptake was lower than caudate nucleus uptake, as previously reported by other ${ }^{123}$ I-FP-CIT studies and in accordance with Spiegel's data.

The occurrence of a widespread degeneration of the nigrostriatal dopaminergic pathway for the development of parkinsonian tremor at rest has been previously postulated by Isaias et al (24), who evaluated 20 drug-naïve PD patients, 10 with unilateral akinesia-rigidity at onset and 10 with additional tremor at rest. They reported a mean reduction of ${ }^{123}$ I-FP-CIT striatal binding of 42 and $50 \%$ for ART and TDT patients, respectively. In particular, there was a significantly lower uptake of ${ }^{123}$ I-FP-CIT in TDT patients when compared to ART ones for both ipsilateral striatum and caudate nucleus.

The results of Isaias et al (24) clearly contrast with our findings and with those of Spiegel et al (15). The reason for this significantly differing striatal ${ }^{123}$ I-FP-CIT uptake in the subtypes of PD remains yet unclear. However, it is worth noting that a recent paper has confirmed the lower ${ }^{123} \mathrm{I}$-FP-CIT striatal binding in ART compared to TDT (25). Neverthless, in the present study differences in nigrostriatal impairment in the clinical variants of early PD were found by SPECT only in the putamen contralateral to the most clinically affected side that showed a lower ${ }^{123}$ I-FP-CIT uptake in ART compared to TDT patients. In fact, no statistically significant differences emerged when considering uptake in the bilateral caudate and ipsilateral putamen.

Postmortem studies in PD indicated that the ART show more severe cell loss in the ventrolateral part of the substantia nigra zona compacta (SNZC), which projects to the dorsal putamen than the medial part projecting to the caudate nucleus and the anterior putamen, with negative correlation between SNZC cell counts, severity of akinesia-rigidity, and dopamine loss in the posterior putamen (26). On the contrary, the TDT shows more severe neuron loss in medial than in lateral SNZC, and in the retrobulbar field, that projects to the dorsolateral striatum and ventromedial thalamus.

On the basis of these neuropathological findings, a retrospective analysis of consecutive ${ }^{123}$ I-FP-CIT SPECT acquired over 3 years was conducted to verify if the PD subtypes and the visual findings of the scintigraphic imaging show a relation in accordance with the Jellinger model, hypothesizing that the 
ART may present with a more pronounced posterior reduction of radiopharmaceutical uptake than the TDT (27). The visual analysis showed a clear association of TDT with an almost normal symmetrical uptake with only a discrete reduction in one or both putamina ('eagle-wing' striatal configuration), and ART with a significant bilateral reduced uptake in the putamen and normal or almost normal uptake in the caudate nuclei ('egg-shaped' striatum). Moreover, a significantly higher ${ }^{123}$ I-FP-CIT uptake was observed in the TDT compared to the ART both for the semi-quantitative and the automatized three-dimensional analysis ipsilateral and contralateral to the most affected side in the putamen and caudate. These outcomes suggest that the visual evaluation of ${ }^{123}$ I-FP-CIT imaging allows for a pattern classification that might reflect the different neuropathological features associated with the different IPD subgroups.

In IPD it has been reported that DAT availability in the putamen mainly correlates with motor function, whereas DAT availability is correlated with prefrontal cognitive functions, particularly in the caudate region (28). Moreover, ${ }^{123}$ I-FP-CIT striatal uptake values significantly correlate with bradykinesia and rigidity but not with tremor $(21,25,29)$. Defining subgroups of patients with IPD can be useful to delineate the natural history, prognosis, and treatment options, especially the optimal therapy for the specific motor and cognitive symptoms. Therefore, the early identification of different patient subtypes by means of a non-invasive molecular imaging technique like ${ }^{123}$ I-FP-CIT SPECT may be important for management strategies.

In particular, a strong association between a non-tremor dominant disease pattern and cognitive disability has been recently found, which is most pronounced in the performance of executive functions; however, the decline in cognitive function in patients with IPD is also influenced by the impairment of non-dopaminergic systems, such as noradrenergic, cholinergic and serotonergic systems $(30,31)$. Moreover, the non-tremor dominant group had a significantly higher mean pathological grading of cortical Lewy bodies and more cortical amyloid- $\beta$ plaques and cerebral amyloid angiopathy than the tremor-dominant patients, and also exhibited a generally more rapid disease progression together with higher scores on the Beck depression inventory, indicating the severity of depressive symptoms.

In conclusion, the results of our study show that in the early stage of IPD the dopaminergic system is more severely impaired in ART than in the other subgroups of patients, in particular when compared to TDT. Moreover, our findings suggest that other neurotransmitter pathways apart from the nigrostriatal dopaminergic pathway are involved in the generation of tremor in IPD; they are consistent with previous evidence of a lack of correlation between ${ }^{123}$ I-FP-CIT uptake and tremor severity. As a matter of fact, in contrast to the other movement symptoms of IPD, tremor is caused by separate mechanisms and is anatomically different from rigidity and bradykinesia $(32,33)$. Therefore, resting tremor is comparatively less influenced by dopamine than other motor symptoms, and the evidence of lesser efficacy of dopaminergic therapy on IPD tremor than on other main symptoms emphasizes the contribution of non-dopaminergic mechanisms. Finally, the relative putamen sparing in TDT could partially explain the slower disease progression reported in this IPD subgroup, and the non-invasive information provided by ${ }^{123}$ I-FP-CIT molecular imaging may help to improve further therapeutic planning.

\section{References}

1. Marsden CA: Dopamine: the rewarding years. Br J Pharmacol 147: 136-144, 2006.

2. Gelb DJ, Oliver E and Gilman S: Diagnostic criteria for Parkinson disease. Arch Neurol 56: 33-39, 1999.

3. Brooks DJ, Ibanez V, Sawle GV, et al: Differing patterns of striatal ${ }^{18} \mathrm{~F}$-dopa uptake in Parkinson's disease, multiple system atrophy and progressive supranuclear palsy. Ann Neurol 28: 547-555, 1990.

4. Davis MR, Votaw JR, Bremner JD, et al: Initial human PET imaging studies with the dopamine transporter ligand ${ }^{18} \mathrm{~F}$-FECNT. J Nucl Med 44: 855-861, 2003.

5. Filippi L,Manni C, Pierantozzi M, et al: ${ }^{123}$ I-FP-CIT in progressive supranuclear palsy and in Parkinson's disease: a SPECT semiquantitative study. Nucl Med Commun 27: 381-386, 2006.

6. Marek K, Jennings D and Seibyl J: Single-photon emission tomography and dopamine transporter imaging in Parkinson's disease. Adv Neurol 91: 183-191, 2003.

7. Ciliax BJ, Heilman C, Demchyshyn LL, et al: The dopamine transporter: immunochemical characterization and localization in brain. J Neurosci 15: 1714-1723, 1995.

8. Ahlskog JE: Diagnosis and differential diagnosis of Parkinson's disease and parkinsonism. Parkinsonism Relat Disord 7: 63-70, 2000.

9. Quinn N: Parkinsonism-recognition and differential diagnosis. BMJ 310: 447-452, 1995.

10. Rajput AH, Voll A, Rajputet ML, et al: Course in Parkinson disease subtypes: a 39-year clinicopathologic study. Neurology 73: 206-212, 2009.

11. Hughes A, Daniel SE, Kilford L and Lees AJ: Accuracy of clinical diagnosis of idiopathic Parkinson's disease: a clinico-pathological study of 100 cases. J Neurol Neurosurg Psychiatry 55: 181-184, 1992.

12. Fahn S, Elton RL and Members of the UPDRS Development Committee: Unified Parkinson's Disease rating scale. In: Recent Development in Parkinson's Disease. Vol. 2. Fahn S, Marsden CD, Calne DB and Goldstein M (eds). Macmillan Healthcare Information, Florhan Park, pp15 3-164, 1987.

13. Kang GA, Bronstein JM, Masterman DL, et al: Clinical characteristics in early Parkinson's disease in a central California population-based study. Mov Disord 20: 1133-1142, 2005.

14. Lewis SJ, Foltynie T, Blackwell AD, Robbins TW, Owen AM and Barker RA: Heterogeneity of Parkinson's disease in the early clinical stages using a data driven approach. J Neurol Neurosurg Psychiatry 76: 343-348, 2005.

15. Spiegel J, Hellwing D, Samnick S, Jost W, Mollers M, Fassbender K, Kirsch CM and Dillman U: Striatal FP-CIT uptake differs in the subtypes of early Parkinson's disease. J Neural Tansm 114: 331-335, 2007.

16. Tatsch K, Asenbaum S, Bartenstein P, et al: European Association of Nuclear Medicine procedure guidelines for brain neurotransmission SPET using ${ }^{123}$ I-labelled dopamine transporter ligands. Eur J Nucl Med Mol Imaging 29: 30-35, 2002.

17. Chang LT: A method for attenuation correction in radionuclide computed tomography. IEEE Trans Nucl Sci NS 25: 638-643, 1978.

18. Booij J, Tissingh G, Winogrodzka A, et al: Practical benefit of ${ }^{123}$ I-FP-CIT SPET in the demonstration of the dopaminergic deficit in Parkinson's disease. Eur J Nucl Med 24: 68-71, 1997.

19. Schillaci O, Pierantozzi M, Filippi L, et al: The effect of levodopa therapy on dopamine transporter SPECT imaging with (123) I-FP-CIT in patients with Parkinson's disease. Eur J Nucl Med Mol Imaging 32: 1452-1456, 2005.

20. Bernheimer H, Birkmayer W, Hornykiewicz O, et al: Brain dopamine and the syndromes of Parkinson and Huntington. Clinical, morphological and neurochemical correlations. J Neurol Sci 20: 415-455, 1973.

21. Benamer TS, Patterson J, Grosset DG, et al: Accurate differentiation of parkinsonism and essential tremor using visual assessment of $\left({ }^{123} \mathrm{I}\right)$-FP-CIT SPECT imaging: the $\left({ }^{123} \mathrm{I}\right)$-FP-CIT study group. Mov Disord 15: 503-510, 2000. 
22. Rajput AH, Sitte HH, Rajput A, et al: Globus pallidus dopamine and Parkinson motor subtypes: clinical and brain biochemical correlation. Neurology 70: 1403-1410, 2008

23. Kägi G, Bhatia KP and Telosa E: The role of DAT-SPECT in movement disorders. J Neurol Neurosurg Psychiatry 81: 5-12, 2010.

24. Isaias IU, Benti R, Cilia R, et al: ${ }^{123}$ I-FP-CIT striatal binding in early Parkinson's disease patients with tremor vs. akinetic-rigid onset. Neuroreport 18: 1499-1502, 2007.

25. Rossi C, Frosini D, Volterrani D, et al: Differences in nigro-striatal impairment in clinical variants of early Parkinson's disease: evidence from a FP-CIT SPECT study. Eur J Neurol 7: 626-630, 2010.

26. Jellinger KA: Post mortem studies on Parkinson's disease: is it possible to detect brain areas for specific symptoms? J Neural Transm Suppl 56: 1-29, 1999.

27. Carsten E, Deniz K, Gereon R, et al: Akinetic-rigid and tremordominant Parkinson's disease patients show different patterns of FP-CIT single photon emission computed tomography. Mov Disord 26: 416-423, 2011
28. Varone A and Halldin C: Molecular imaging of the dopamine transporter. J Nucl Med 51: 1331-1334, 2010.

29. Pirker W: Correlation of dopamine transporter imaging with parkinsonian motor handicap: how close is it? Mov Disord 18: 43-51, 2003.

30. Selikhova M, Williams DR, Kempster PA, Holton JL, Revesz T and Lees AJ: A clinico-pathological study of subtypes in Parkinson's disease. Brain 132: 2947-2957, 2009.

31. Gordon PH, Pullman SL, Louis ED, et al: Mirtazapine in Parkinsonian tremor. Parkinsonism Relat Disord 9: 125-126, 2002.

32. Carr J: Tremor in Parkinson's disease. Parkinsonism Relat Disord 8: 223-234, 2002.

33. Rivlin-Etzion M, Marmor O, Heimer G, Raz A, Nini A and Bergman $\mathrm{H}$ : Basal ganglia oscillations and pathophysiology of movement disorders. Curr Opin Neurobiol 16: 629-637, 2006. 